Research Article

\title{
Convergence Analysis for a System of Equilibrium Problems and a Countable Family of Relatively Quasi-Nonexpansive Mappings in Banach Spaces
}

\author{
Prasit Cholamjiak and Suthep Suantai \\ Department of Mathematics, Faculty of Science, Chiang Mai University, Chiang Mai 50200, Thailand \\ Correspondence should be addressed to Suthep Suantai, scmti005@chiangmai.ac.th
}

Received 11 April 2010; Revised 9 July 2010; Accepted 16 July 2010

Academic Editor: Simeon Reich

Copyright (c) 2010 P. Cholamjiak and S. Suantai. This is an open access article distributed under the Creative Commons Attribution License, which permits unrestricted use, distribution, and reproduction in any medium, provided the original work is properly cited.

We introduce a new hybrid iterative scheme for finding a common element in the solutions set of a system of equilibrium problems and the common fixed points set of an infinitely countable family of relatively quasi-nonexpansive mappings in the framework of Banach spaces. We prove the strong convergence theorem by the shrinking projection method. In addition, the results obtained in this paper can be applied to a system of variational inequality problems and to a system of convex minimization problems in a Banach space.

\section{Introduction}

Let $E$ be a real Banach space, and let $E^{*}$ be the dual of $E$. Let $C$ be a closed and convex subset of $E$. Let $\left\{f_{j}\right\}_{j \in \Lambda}$ be bifunctions from $C \times C$ to $\mathbb{R}$, where $\mathbb{R}$ is the set of real numbers and $\Lambda$ is an arbitrary index set. The system of equilibrium problems is to find $\widehat{x} \in C$ such that

$$
f_{j}(\widehat{x}, \mathrm{y}) \geq 0, \quad \forall y \in C, j \in \Lambda
$$

If $\Lambda$ is a singleton, then problem (1.1) reduces to find $\widehat{x} \in C$ such that

$$
f(\widehat{x}, y) \geq 0, \quad \forall y \in C
$$

The set of solutions of the equilibrium problem (1.2) is denoted by $\operatorname{EP}(f)$. 
Combettes and Hirstoaga [1] introduced an iterative scheme for finding a common element in the solutions set of problem (1.1) in a Hilbert space and obtained a weak convergence theorem.

In 2004, Matsushita and Takahashi [2] introduced the following algorithm for a relatively nonexpansive mapping $T$ in a Banach space $E$ : for any initial point $x_{0} \in C$, define the sequence $\left\{x_{n}\right\}$ by

$$
x_{n+1}=\Pi_{C} J^{-1}\left(\alpha_{n} J x_{n}+\left(1-\alpha_{n}\right) J T x_{n}\right), \quad n \geq 0,
$$

where $J$ is the duality mapping on $E, \Pi_{C}$ is the generalized projection from $E$ onto $C$, and $\left\{\alpha_{n}\right\}$ is a sequence in $[0,1]$. They proved that the sequence $\left\{x_{n}\right\}$ converges weakly to fixed point of $T$ under some suitable conditions on $\left\{\alpha_{n}\right\}$.

In 2008, Takahashi and Zembayashi [3] introduced the following iterative scheme which is called the shrinking projection method for a relatively nonexpansive mapping $T$ and an equilibrium problem in a Banach space $E$ :

$$
\begin{gathered}
x_{0}=x \in C, \quad C_{0}=C, \\
y_{n}=J^{-1}\left(\alpha_{n} J x_{n}+\left(1-\alpha_{n}\right) J T x_{n}\right), \\
u_{n} \in C \text { such that } f\left(u_{n}, y\right)+\frac{1}{r_{n}}\left\langle y-u_{n}, J u_{n}-J y_{n}\right\rangle \geq 0 \quad \forall y \in C, \\
C_{n+1}=\left\{z \in C_{n}: \phi\left(z, u_{n}\right) \leq \phi\left(z, x_{n}\right)\right\}, \\
x_{n+1}=\prod_{C_{n+1}} x_{0}, \quad n \geq 0 .
\end{gathered}
$$

They proved that the sequence $\left\{x_{n}\right\}$ converges strongly to $\Pi_{F(T) \cap E P(f)} x_{0}$ under some appropriate conditions.

\section{Preliminaries and Lemmas}

Let $E$ be a real Banach space, and let $U=\{x \in E:\|x\|=1\}$ be the unit sphere of $E$. A Banach space $E$ is said to be strictly convex if, for any $x, y \in U$,

$$
x \neq y \text { implies }\left\|\frac{x+y}{2}\right\|<1 .
$$

It is also said to be uniformly convex if, for each $\varepsilon \in(0,2]$, there exists $\delta>0$ such that, for any $x, y \in U$,

$$
\|x-y\| \geq \varepsilon \text { implies }\left\|\frac{x+y}{2}\right\|<1-\delta \text {. }
$$


It is known that a uniformly convex Banach space is reflexive and strictly convex. The function $\delta:[0,2] \rightarrow[0,1]$ which is called the modulus of convexity of $E$ is defined as follows:

$$
\delta(\varepsilon)=\inf \left\{1-\left\|\frac{x+y}{2}\right\|: x, y \in E,\|x\|=\|y\|=1,\|x-y\| \geq \varepsilon\right\}
$$

The space $E$ is uniformly convex if and only if $\delta(\varepsilon)>0$ for all $\varepsilon \in(0,2]$. A Banach space $E$ is said to be smooth if the limit

$$
\lim _{t \rightarrow 0} \frac{\|x+t y\|-\|x\|}{t}
$$

exists for all $x, y \in U$. It is also said to be uniformly smooth if the limit (2.4) is attained uniformly for $x, y \in U$. The duality mapping $J: E \rightarrow 2^{E^{*}}$ is defined by

$$
J(x)=\left\{x^{*} \in E^{*}:\left\langle x, x^{*}\right\rangle=\|x\|^{2}=\left\|x^{*}\right\|^{2}\right\}
$$

for all $x \in E$. If $E$ is a Hilbert space, then $J=I$, where $I$ is the identity operator. It is also known that, if $E$ is uniformly smooth, then $J$ is uniformly norm-to-norm continuous on bounded subset of $E$ (see [4] for more details).

Let $E$ be a smooth Banach space. The function $\phi: E \times E \rightarrow \mathbb{R}$ is defined by

$$
\phi(x, y)=\|x\|^{2}-2\langle x, J y\rangle+\|y\|^{2}
$$

for all $x, y \in E$. In a Hilbert space $H$, we have $\phi(x, y)=\|x-y\|^{2}$ for all $x, y \in H$.

Let $C$ be a closed and convex subset of $E$, and let $T$ be a mapping from $C$ into itself. A point $p$ in $C$ is said to be an asymptotic fixed point of $T$ [5] if $C$ contains a sequence $\left\{x_{n}\right\}$ which converges weakly to $p$ such that $\lim _{n \rightarrow \infty}\left\|x_{n}-T x_{n}\right\|=0$. The set of asymptotic fixed points of $T$ will be denoted by $\widehat{F}(T)$. A mapping $T$ is said to be relatively nonexpansive [6-8] if $\widehat{F}(T)=F(T)$ and $\phi(p, T x) \leq \phi(p, x)$ for all $p \in F(T)$ and $x \in C$. The asymptotic behavior of a relatively nonexpansive mapping was studied in [6,7]. $T$ is said to be relatively quasinonexpansive if $F(T) \neq \emptyset$ and $\phi(p, T x) \leq \phi(p, x)$ for all $p \in F(T)$ and $x \in C$. It is obvious that the class of relatively quasi-nonexpansive mappings is more general than the class of relatively nonexpansive mappings. The class of relatively quasi-nonexpansive mappings was studied by many authors (see, for example, [9-12]). Recall that $T$ is closed if

$$
x_{n} \longrightarrow x, \quad T x_{n} \longrightarrow y \text { imply } T x=y
$$

The aim of this paper is to introduce a new hybrid projection algorithm for finding a common element in the solutions set of a system of equilibrium problems and the common fixed points set of an infinitely countable family of closed and relatively quasi-nonexpansive mappings in the frameworks of Banach spaces.

We will need the following lemmas. 
Lemma 2.1 (Kamimura and Takahashi [8]). Let E be a uniformly convex and smooth Banach space, and let $\left\{x_{n}\right\},\left\{y_{n}\right\}$ be two sequences of $E$. If $\phi\left(x_{n}, y_{n}\right) \rightarrow 0$ and either $\left\{x_{n}\right\}$ or $\left\{y_{n}\right\}$ is bounded, then $\left\|x_{n}-y_{n}\right\| \rightarrow 0$ as $n \rightarrow \infty$.

Let $E$ be a reflexive, strictly convex, and smooth Banach space, and let $C$ be a nonempty, closed, and convex subset of $E$. The generalized projection mapping, introduced by Alber [13], is a mapping $\Pi_{C}: E \rightarrow C$ that assigns to an arbitrary point $x \in E$ the minimum point of the function $\phi(y, x)$; that is, $\Pi_{C} x=\bar{x}$, where $\bar{x}$ is the solution of the minimization problem

$$
\phi(\bar{x}, x)=\min \{\phi(y, x): y \in C\}
$$

The existence and uniqueness of the operator $\Pi_{C}$ follows from the properties of the functional $\phi$ and strict monotonicity of the duality mapping $J$ (see, for instance, $[4,8,13-15]$ ). In a Hilbert space, $\Pi_{C}$ is coincident with the metric projection.

Lemma 2.2 (Alber [13], Kamimura and Takahashi [8]). Let $C$ be a nonempty, closed, and convex subset of a smooth, strictly convex, and reflexive Banach space $E$, let $x \in E$, and let $z \in C$. Then $z=\Pi_{C} x$ if and only if

$$
\langle y-z, J x-J z\rangle \leq 0, \quad \forall y \in C
$$

Lemma 2.3 (Alber [13], Kamimura and Takahashi [8]). Let $C$ be a nonempty, closed, and convex subset of a smooth, strictly convex, and reflexive Banach space E. Then

$$
\phi\left(x, \Pi_{C} y\right)+\phi\left(\Pi_{C} y, y\right) \leq \phi(x, y) \quad \forall x \in C, y \in E .
$$

Lemma 2.4 (Qin et al. [16]). Let E be a uniformly convex, smooth Banach space, and let $C$ be a closed and convex subset of $E$. Let $T$ be a closed and relatively quasi-nonexpansive mapping from $C$ into itself. Then $F(T)$ is closed and convex.

For solving the equilibrium problem, let us assume that a bifunction $f$ satisfies the following conditions:

(A1) $f(x, x)=0$ for all $x \in C$;

(A2) $f$ is monotone; that is, $f(x, y)+f(y, x) \leq 0$ for all $x, y \in C$;

(A3) for all $x, y, z \in C, \lim \sup _{t \downarrow} f(t z+(1-t) x, y) \leq f(x, y)$;

(A4) for all $x \in C, f(x, \cdot)$ is convex and lower semicontinuous.

Lemma 2.5 (Blum and Oettli [17]). Let $C$ be a closed and convex subset of a smooth, strictly convex, and reflexive Banach space $E$, let $f$ be a bifunction from $C \times C$ to $\mathbb{R}$ which satisfies conditions (A1)(A4), and let $r>0$ and $x \in E$. Then there exists $z \in C$ such that

$$
f(z, y)+\frac{1}{r}\langle y-z, J z-J x\rangle \geq 0, \quad \forall y \in C .
$$


Lemma 2.6 (Takahashi and Zembayashi [18]). Let C be a closed and convex subset of a uniformly smooth, strictly convex, and reflexive Banach space $E$, and let $f$ be a bifunction from $C \times C$ to $\mathbb{R}$ which satisfies conditions (A1)-(A4). For all $r>0$ and $x \in E$, define the mapping $T_{r}^{f}: E \rightarrow C$ as follows:

$$
T_{r}^{f}(x)=\left\{z \in C: f(z, y)+\frac{1}{r}\langle y-z, J z-J x\rangle \geq 0, \forall y \in C\right\}
$$

Then, the following statements hold:

(1) $T_{r}^{f}$ is single valued;

(2) $T_{r}^{f}$ is of firmly nonexpansive type [19]; that is, for all $x, y \in E$,

$$
\left\langle T_{r}^{f} x-T_{r}^{f} y, J T_{r}^{f} x-J T_{r}^{f} y\right\rangle \leq\left\langle T_{r}^{f} x-T_{r}^{f} y, J x-J y\right\rangle
$$

(3) $F\left(T_{r}^{f}\right)=E P(f)$;

(4) $E P(f)$ is closed and convex.

Lemma 2.7 (Takahashi and Zembayashi [18]). Let $C$ be a closed and convex subset of a smooth, strictly, and reflexive Banach space $E$, let $f$ be a bifunction from $C \times C$ to $\mathbb{R}$ which satisfies conditions (A1)-(A4), and let $r>0$. Then, for all $x \in E$ and $q \in F\left(T_{r}^{f}\right)$,

$$
\phi\left(q, T_{r}^{f} x\right)+\phi\left(T_{r}^{f} x, x\right) \leq \phi(q, x) .
$$

\section{Strong Convergence Theorems}

Theorem 3.1. Let $E$ be a uniformly convex and uniformly smooth Banach space, and let $C$ be a nonempty, closed, and convex subset of $E$. Let $\left\{f_{j}\right\}_{j=1}^{M}$ be bifunctions from $C \times C$ to $\mathbb{R}$ which satisfies conditions (A1)-(A4), and let $\left\{T_{i}\right\}_{i=1}^{\infty}$ be an infinitely countable family of closed and relatively quasinonexpansive mappings from $C$ into itself. Assume that $F:=\left(\bigcap_{i=1}^{\infty} F\left(T_{i}\right)\right) \cap\left(\bigcap_{j=1}^{M} E P\left(f_{j}\right)\right) \neq \emptyset$. For any initial point $x_{0} \in E$ with $x_{1}=\Pi_{C_{1}} x_{0}$ and $C_{1}=C$, define the sequence $\left\{x_{n}\right\}$ as follows:

$$
\begin{gathered}
y_{n, i}=J^{-1}\left(\alpha_{n} J x_{n}+\left(1-\alpha_{n}\right) J T_{i} x_{n}\right), \\
u_{n, i}=T_{r_{M, n}}^{f_{M}} T_{r_{M-1, n}}^{f_{M-1}} \cdots T_{r_{1, n}}^{f_{1}} y_{n, i}, \\
C_{n+1}=\left\{z \in C_{n}: \sup _{i \geq 1} \phi\left(z, u_{n, i}\right) \leq \phi\left(z, x_{n}\right)\right\}, \\
x_{n+1}=\prod_{C_{n+1}} x_{0}, \quad n \geq 1 .
\end{gathered}
$$

Assume that $\left\{\alpha_{n}\right\}$ and $\left\{r_{j, n}\right\}$ for $j=1,2, \ldots, M$ are sequences which satisfy the following conditions:

(B1) $\lim \sup _{n \rightarrow \infty} \alpha_{n}<1$;

(B2) $\lim \inf _{n \rightarrow \infty} r_{j, n}>0$.

Then the sequence $\left\{x_{n}\right\}$ converges strongly to $\Pi_{F} x_{0}$. 
Proof. We divide our proof into six steps as follows.

Step 1. $F \subset C_{n}$ for all $n \geq 1$.

From Lemma 2.4 we know that $F\left(T_{i}\right)$ is closed, and convex for all $i \geq 1$. From Lemma 2.6(4), we also know that $\operatorname{EP}\left(f_{j}\right)$ is closed and convex for each $j=1,2, \ldots, M$. Hence $F:=\left(\bigcap_{i=1}^{\infty} F\left(T_{i}\right)\right) \cap\left(\bigcap_{j=1}^{M} \operatorname{EP}\left(f_{j}\right)\right)$ is a nonempty, closed and convex subset of $C$. Clearly $C_{1}=C$ is closed and convex. Suppose that $C_{k}$ is closed and convex for some $k \in \mathbb{N}$. For each $z \in C_{k}$ and $i \geq 1$, we see that $\phi\left(z, u_{k, i}\right) \leq \phi\left(z, x_{k}\right)$ is equivalent to

$$
2\left\langle z, J x_{k}\right\rangle-2\left\langle z, J u_{k, i}\right\rangle \leq\left\|x_{k}\right\|^{2}-\left\|u_{k, i}\right\|^{2} .
$$

By the construction of the set $C_{k+1}$, we see that

$$
\begin{aligned}
C_{k+1} & =\left\{z \in C_{k}: \sup _{i \geq 1} \phi\left(z, u_{k, i}\right) \leq \phi\left(z, x_{k}\right)\right\} \\
& =\bigcap_{i=1}^{\infty}\left\{z \in C_{k}: \phi\left(z, u_{k, i}\right) \leq \phi\left(z, x_{k}\right)\right\} .
\end{aligned}
$$

Hence $C_{k+1}$ is also closed and convex.

It is obvious that $F \subset C_{1}=C$. Now, suppose that $F \subset C_{k}$ for some $k \in \mathbb{N}$, and let $p \in F:=\left(\bigcap_{i=1}^{\infty} F\left(T_{i}\right)\right) \cap\left(\bigcap_{j=1}^{M} \operatorname{EP}\left(f_{j}\right)\right)$. Then

$$
\begin{aligned}
\phi\left(p, u_{k, i}\right)= & \phi\left(p, T_{r_{M, n}}^{f_{M}} T_{r_{M-1, n}}^{f_{M-1}} \cdots T_{r_{1, n}}^{f_{1}} y_{k, i}\right) \\
\leq & \phi\left(p, T_{r_{M-1, n}}^{f_{M-1}} T_{r_{M-2, n}}^{f_{M-2}} \cdots T_{r_{1, n}}^{f_{1}} y_{k, i}\right) \\
\vdots & \leq \phi\left(p, T_{r_{1, n}}^{f_{1}} y_{k, i}\right) \\
\leq & \phi\left(p, y_{k, i}\right) \\
= & \phi\left(p, J^{-1}\left(\alpha_{k} J x_{k}+\left(1-\alpha_{k}\right) J T_{i} x_{k}\right)\right) \\
= & \|p\|^{2}-2\left\langle p, \alpha_{k} J x_{k}+\left(1-\alpha_{k}\right) J T_{i} x_{k}\right\rangle \\
& +\left\|\alpha_{k} J x_{k}+\left(1-\alpha_{k}\right) J T_{i} x_{k}\right\|^{2} \\
\leq & \|p\|^{2}-2 \alpha_{k}\left\langle p, J x_{k}\right\rangle-2\left(1-\alpha_{k}\right)\left\langle p, J T_{i} x_{k}\right\rangle \\
& +\alpha_{k}\left\|x_{k}\right\|^{2}+\left(1-\alpha_{k}\right)\left\|T_{i} x_{k}\right\|^{2} \\
= & \alpha_{k} \phi\left(p, x_{k}\right)+\left(1-\alpha_{k}\right) \phi\left(p, T_{i} x_{k}\right) \\
\leq & \phi\left(p, x_{k}\right) .
\end{aligned}
$$

Hence $F \subset C_{k+1}$. By induction, we can conclude that $F \subset C_{n}$ for all $n \geq 1$. 
Step 2. $\lim _{n \rightarrow \infty} \phi\left(x_{n}, x_{0}\right)$ exists.

From $x_{n}=\Pi_{C_{n}} x_{0}$ and $x_{n+1}=\prod_{C_{n+1}} x_{0} \in C_{n+1} \subset C_{n}$, we have

$$
\phi\left(x_{n}, x_{0}\right) \leq \phi\left(x_{n+1}, x_{0}\right), \quad n \geq 1
$$

From Lemma 2.3 we get that

$$
\phi\left(x_{n}, x_{0}\right)=\phi\left(\Pi_{C_{n}} x_{0}, x_{0}\right) \leq \phi\left(p, x_{0}\right)-\phi\left(p, x_{n}\right) \leq \phi\left(p, x_{0}\right) .
$$

Combining (3.5) and (3.6), we get that $\lim _{n \rightarrow \infty} \phi\left(x_{n}, x_{0}\right)$ exists.

Step 3. $\left\{x_{n}\right\}$ is a Cauchy sequence in $C$.

Since $x_{m}=\Pi_{C_{m}} x_{0} \in C_{m} \subset C_{n}$ for $m>n$, we obtain from Lemma 2.3 that

$$
\begin{aligned}
\phi\left(x_{m}, x_{n}\right)=\phi\left(x_{m}, \Pi_{C_{n}} x_{0}\right) & \leq \phi\left(x_{m}, x_{0}\right)-\phi\left(\Pi_{C_{n}} x_{0}, x_{0}\right) \\
& =\phi\left(x_{m}, x_{0}\right)-\phi\left(x_{n}, x_{0}\right) .
\end{aligned}
$$

We see that $\phi\left(x_{m}, x_{n}\right) \rightarrow 0$ as $m, n \rightarrow \infty$, which implies with Lemma 2.1 that $\left\|x_{m}-x_{n}\right\| \rightarrow 0$ as $m, n \rightarrow \infty$. Therefore $\left\{x_{n}\right\}$ is a Cauchy sequence. By the completeness of the space $E$ and the closedness of the set $C$, we can assume that $x_{n} \rightarrow q \in C$ as $n \rightarrow \infty$. Moreover, we get that

$$
\lim _{n \rightarrow \infty} \phi\left(x_{n+1}, x_{n}\right)=0
$$

Since $x_{n+1}=\Pi_{C_{n+1}} x_{0} \in C_{n+1}$, we have for all $i \geq 1$ that

$$
\phi\left(x_{n+1}, u_{n, i}\right) \leq \phi\left(x_{n+1}, x_{n}\right) \longrightarrow 0
$$

Applying Lemma 2.1 to (3.8) and (3.9), we derive

$$
\lim _{n \rightarrow \infty}\left\|u_{n, i}-x_{n}\right\|=0, \quad \forall i \geq 1
$$

This shows that $u_{n, i} \rightarrow q$ as $n \rightarrow \infty$ for all $i \geq 1$. Since $J$ is uniformly norm-to-norm continuous on bounded subsets of $E$, we obtain that

$$
\lim _{n \rightarrow \infty}\left\|J u_{n, i}-J x_{n}\right\|=0, \quad \forall i \geq 1
$$

Step 4. $q \in \bigcap_{i=1}^{\infty} F\left(T_{i}\right)$.

Denote $\Theta_{n}^{j}=T_{r_{j, n}}^{f_{j}} T_{r_{j-1, n}}^{f_{j-1}} \cdots T_{r_{1, n}}^{f_{1}}$ for any $j \in\{1,2, \ldots, M\}$ and $\Theta_{n}^{0}=I$ for all $n \geq 1$. We note that $u_{n, i}=\Theta_{n}^{M} y_{n, i}$ for all $i \geq 1$. From (3.4) we observe that

$$
\phi\left(p, \Theta_{n}^{M-1} y_{n, i}\right) \leq \phi\left(p, \Theta_{n}^{M-2} y_{n, i}\right) \leq \cdots \leq \phi\left(p, y_{n, i}\right) \leq \phi\left(p, x_{n}\right), \quad \forall i \geq 1 .
$$


Since $p \in \operatorname{EP}\left(f_{M}\right)=F\left(T_{r_{M, n}}^{f_{M}}\right)$ for all $n \geq 1$, it follows from (3.12) and Lemma 2.7 that

$$
\begin{aligned}
\phi\left(u_{n, i}, \Theta_{n}^{M-1} y_{n, i}\right) & \leq \phi\left(p, \Theta_{n}^{M-1} y_{n, i}\right)-\phi\left(p, u_{n, i}\right) \\
& \leq \phi\left(p, x_{n}\right)-\phi\left(p, u_{n, i}\right) .
\end{aligned}
$$

From (3.10) and (3.11), we get that $\lim _{n \rightarrow \infty} \phi\left(u_{n, i}, \Theta_{n}^{M-1} y_{n, i}\right)=0$ for all $i \geq 1$. From Lemma 2.1, we have

$$
\lim _{n \rightarrow \infty}\left\|u_{n, i}-\Theta_{n}^{M-1} y_{n, i}\right\|=0, \quad \forall i \geq 1
$$

From (3.10) and (3.14), we have

$$
\lim _{n \rightarrow \infty}\left\|x_{n}-\Theta_{n}^{M-1} y_{n, i}\right\|=0, \quad \forall i \geq 1
$$

and hence,

$$
\lim _{n \rightarrow \infty}\left\|J x_{n}-J \Theta_{n}^{M-1} y_{n, i}\right\|=0, \quad \forall i \geq 1
$$

Again, since $p \in \operatorname{EP}\left(f_{M-1}\right)=F\left(T_{r_{M-1, n}}^{f_{M-1}}\right)$ for all $n \geq 1$, it follows from (3.12) and Lemma 2.7 that

$$
\begin{aligned}
\phi\left(\Theta_{n}^{M-1} y_{n, i}, \Theta_{n}^{M-2} y_{n, i}\right) & \leq \phi\left(p, \Theta_{n}^{M-2} y_{n, i}\right)-\phi\left(p, \Theta_{n}^{M-1} y_{n, i}\right) \\
& \leq \phi\left(p, x_{n}\right)-\phi\left(p, \Theta_{n}^{M-1} y_{n, i}\right) .
\end{aligned}
$$

From (3.15) and (3.16), we also have

$$
\lim _{n \rightarrow \infty}\left\|\Theta_{n}^{M-1} y_{n, i}-\Theta_{n}^{M-2} y_{n, i}\right\|=0, \quad \forall i \geq 1
$$

Hence, from (3.15) and (3.18), we get

$$
\begin{gathered}
\lim _{n \rightarrow \infty}\left\|x_{n}-\Theta_{n}^{M-2} y_{n, i}\right\|=0, \quad \forall i \geq 1, \\
\lim _{n \rightarrow \infty}\left\|J x_{n}-J \Theta_{n}^{M-2} y_{n, i}\right\|=0, \quad \forall i \geq 1 .
\end{gathered}
$$

In a similar way, we can verify that

$$
\lim _{n \rightarrow \infty}\left\|\Theta_{n}^{M-2} y_{n, i}-\Theta_{n}^{M-3} y_{n, i}\right\|=\cdots=\lim _{n \rightarrow \infty}\left\|\Theta_{n}^{1} y_{n, i}-y_{n, i}\right\|=0
$$


for all $i \geq 1$,

$$
\lim _{n \rightarrow \infty}\left\|x_{n}-\Theta_{n}^{M-3} y_{n, i}\right\|=\cdots=\lim _{n \rightarrow \infty}\left\|x_{n}-y_{n, i}\right\|=0
$$

for all $i \geq 1$,

$$
\lim _{n \rightarrow \infty}\left\|J x_{n}-J \Theta_{n}^{M-3} y_{n, i}\right\|=\cdots=\lim _{n \rightarrow \infty}\left\|J x_{n}-J y_{n, i}\right\|=0
$$

for all $i \geq 1$. Hence, we can conclude that

$$
\lim _{n \rightarrow \infty}\left\|\Theta_{n}^{j} y_{n, i}-\Theta_{n}^{j-1} y_{n, i}\right\|=0
$$

for each $j=1,2, \ldots, M$ and $i \geq 1$. Observe that

$$
\begin{aligned}
\left\|J y_{n, i}-J x_{n}\right\| & =\left\|\alpha_{n} J x_{n}+\left(1-\alpha_{n}\right) J T_{i} x_{n}-J x_{n}\right\| \\
& =\left(1-\alpha_{n}\right)\left\|J T_{i} x_{n}-J x_{n}\right\|
\end{aligned}
$$

then we obtain from (B1) and (3.23) that

$$
\lim _{n \rightarrow \infty}\left\|J T_{i} x_{n}-J x_{n}\right\|=0, \quad \forall i \geq 1
$$

Since $J^{-1}$ is also uniformly norm-to-norm continuous on bounded subsets, we get that

$$
\lim _{n \rightarrow \infty}\left\|T_{i} x_{n}-x_{n}\right\|=0, \quad \forall i \geq 1
$$

Since $T_{i}$ is closed for all $i \geq 1$ and $x_{n} \rightarrow q$, we conclude that $q \in \bigcap_{i=1}^{\infty} F\left(T_{i}\right)$.

Step 5. $q \in \bigcap_{j=1}^{M} \operatorname{EP}\left(f_{j}\right)$.

From (3.24) and (B2), we have that $\left\|J \Theta_{n}^{j} y_{n, i}-J \Theta_{n}^{j-1} y_{n, i}\right\| / r_{j, n} \rightarrow 0$ as $n \rightarrow \infty$. Then, for each $j=1,2, \ldots, M$, we obtain that

$$
f_{j}\left(\Theta_{n}^{j} y_{n, i}, y\right)+\frac{1}{r_{j, n}}\left\langle y-\Theta_{n}^{j} y_{n, i}, J \Theta_{n}^{j} y_{n, i}-J \Theta_{n}^{j-1} y_{n, i}\right\rangle \geq 0, \quad \forall y \in C
$$

From (A2) we have that

$$
\begin{aligned}
\left\|y-\Theta_{n}^{j} y_{n, i}\right\| \frac{\left\|\Theta_{n}^{j} y_{n, i}-J \Theta_{n}^{j-1} y_{n, i}\right\|}{r_{j, n}} & \geq \frac{1}{r_{j, n}}\left\langle y-\Theta_{n}^{j} y_{n, i}, J \Theta_{n}^{j} y_{n, i}-J \Theta_{n}^{j-1} y_{n, i}\right\rangle \\
& \geq-f_{j}\left(\Theta_{n}^{j} y_{n, i}, y\right) \geq f_{j}\left(y_{,} \Theta_{n}^{j} y_{n, i}\right), \quad \forall y \in C .
\end{aligned}
$$


From (A4) and the fact that $\Theta_{n}^{j} y_{n, i} \rightarrow q$ for $i \geq 1$, we get $f_{j}(y, q) \leq 0$ for all $y \in C$. For each $0<t<1$ and $y \in C$, denote $y_{t}=t y+(1-t) q$. Then $y_{t} \in C$, which implies that $f_{j}\left(y_{t}, q\right) \leq 0$. From (A1) and (A4), we obtain that $0=f_{j}\left(y_{t}, y_{t}\right) \leq t f_{j}\left(y_{t}, y\right)+(1-t) f_{j}\left(y_{t}, q\right) \leq t f_{j}\left(y_{t}, y\right)$. Thus, $f_{j}\left(y_{t}, y\right) \geq 0$. From (A3), we have $f_{j}(q, y) \geq 0$ for all $y \in C$ and $j=1,2, \ldots, M$. Hence $q \in \bigcap_{j=1}^{M} \mathrm{EP}\left(f_{j}\right)$.

Step 6. $q=\Pi_{F} x_{0}$.

From $x_{n}=\Pi_{C_{n}} x_{0}$, we have

$$
\left\langle J x_{0}-J x_{n}, x_{n}-z\right\rangle \geq 0 \quad \forall z \in C_{n} .
$$

Since $F \subset C_{n}$, we also have

$$
\left\langle J x_{0}-J x_{n}, x_{n}-p\right\rangle \geq 0 \quad \forall p \in F
$$

Letting $n \rightarrow \infty$ in (3.31), we obtain that

$$
\left\langle J x_{0}-J q, q-p\right\rangle \geq 0 \quad \forall p \in F
$$

From Lemma 2.2 we conclude that $q=\Pi_{F} x_{0}$. This completes the proof.

Remark 3.2. Theorem 3.1 improves and extends Theorem 3.1 of Takahashi and Zembayashi in [3] in the following senses:

(i) from the case of an equilibrium problem to a finite family of equilibrium problems;

(ii) from a single relatively nonexpansive mapping to an infinitely countable family of relatively quasi-nonexpansive mappings;

(iii) if $M=1$ and $T_{i}=T$ for all $i \geq 1$, then our restriction on $\left\{\alpha_{n}\right\}$ is weaker than that of Theorem 3.1 of [3].

Remark 3.3. The iteration (3.1) is a modification of (1.4) in the following ways.

(i) We use the composition of mappings $\left\{T_{r_{j, n}}^{f_{j}}\right\}_{j=1}^{M}$ in the second step.

(ii) We construct the set $C_{n+1}$ by using the concept of supremum concerning an infinitely countable family of closed and relatively quasi-nonexpansive mappings $\left\{T_{i}\right\}_{i=1}^{\infty}$. If $M=1$ and $T_{i}=T$ for all $i \geq 1$, then the iteration (3.1) reduces to that of (1.4).

If we take $\alpha_{n}=0$ for all $n \in \mathbb{N}$ in Theorem 3.1, then we have the following corollary.

Corollary 3.4. Let $E$ be a uniformly convex and uniformly smooth Banach space, and let $C$ be a nonempty, closed, and convex subset of $E$. Let $\left\{f_{j}\right\}_{j=1}^{M}$ be bifunctions from $C \times C$ to $\mathbb{R}$ which satisfies 
conditions (A1)-(A4), and let $\left\{T_{i}\right\}_{i=1}^{\infty}$ be an infinitely countable family of closed and relatively quasinonexpansive mappings from $C$ into itself. Assume that $F:=\left(\bigcap_{i=1}^{\infty} F\left(T_{i}\right)\right) \cap\left(\bigcap_{j=1}^{M} E P\left(f_{j}\right)\right) \neq \emptyset$. For any initial point $x_{0} \in E$ with $x_{1}=\Pi_{C_{1}} x_{0}$ and $C_{1}=C$, define the sequence $\left\{x_{n}\right\}$ as follows:

$$
\begin{gathered}
y_{n, i}=T_{i} x_{n}, \\
u_{n, i}=T_{r_{M, n}}^{f_{M}} T_{r_{M-1, n}}^{f_{M-1}} \cdots T_{r_{1, n}}^{f_{1}} y_{n, i}, \\
C_{n+1}=\left\{z \in C_{n}: \sup _{i \geq 1} \phi\left(z, u_{n, i}\right) \leq \phi\left(z, x_{n}\right)\right\}, \\
x_{n+1}=\prod_{C_{n+1}} x_{0}, \quad n \geq 1 .
\end{gathered}
$$

If $\lim \inf _{n \rightarrow \infty} r_{j, n}>0$ for each $j=1,2, \ldots, M$, then $\left\{x_{n}\right\}$ converges strongly to $\Pi_{F} x_{0}$.

\section{Applications}

In this section, we give several applications of Theorem 3.1 in the framework of Banach spaces and Hilbert spaces.

Let $A: C \rightarrow E^{*}$ be a nonlinear mapping. The classical variational inequality problem is to find that $\widehat{x} \in C$ such that

$$
\langle A \widehat{x}, y-\widehat{x}\rangle \geq 0 \quad \forall y \in C
$$

The solutions set of (4.1) is denoted by $V I(C, A)$. For each $r>0$ and $x \in E$, define the mapping $T_{r}^{A}: E \rightarrow C$ as follows:

$$
T_{r}^{A}(x)=\left\{z \in C:\langle A z, y-z\rangle+\frac{1}{r}\langle y-z, J z-J x\rangle \geq 0, \forall y \in C\right\}
$$

Theorem 4.1. Let $E$ be a uniformly convex and uniformly smooth Banach space, and let $C$ be a nonempty, closed, and convex subset of $E$. Let $\left\{A_{j}\right\}_{j=1}^{M}$ be continuous and monotone operators from $C$ to $E^{*}$, and let $\left\{T_{i}\right\}_{i=1}^{\infty}$ be an infinitely countable family of closed and relatively quasi-nonexpansive mappings from $C$ into itself such that $F:=\left(\bigcap_{i=1}^{\infty} F\left(T_{i}\right)\right) \cap\left(\bigcap_{j=1}^{M} V I\left(C, A_{j}\right)\right) \neq \emptyset$. For any initial point $x_{0} \in E$ with $x_{1}=\Pi_{C_{1}} x_{0}$ and $C_{1}=C$, define the sequence $\left\{x_{n}\right\}$ as follows:

$$
\begin{gathered}
y_{n, i}=J^{-1}\left(\alpha_{n} J x_{n}+\left(1-\alpha_{n}\right) J T_{i} x_{n}\right), \\
u_{n, i}=T_{r_{M, n}}^{A_{M}} T_{r_{M-1, n}}^{A_{M-1}} \cdots T_{r_{1, n}}^{A_{1}} y_{n, i}, \\
C_{n+1}=\left\{z \in C_{n}: \sup _{i \geq 1} \phi\left(z, u_{n, i}\right) \leq \phi\left(z, x_{n}\right)\right\}, \\
x_{n+1}=\prod_{C_{n+1}} x_{0}, \quad n \geq 1 .
\end{gathered}
$$


Assume that $\left\{\alpha_{n}\right\}$ and $\left\{r_{j, n}\right\}$ for $j=1,2, \ldots, M$ are sequences which satisfy conditions (B1) and (B2) of Theorem 3.1.

Then the sequence $\left\{x_{n}\right\}$ converges strongly to $\Pi_{F} x_{0}$.

Proof. Define $f_{j}(x, y)=\left\langle A_{j} x, y-x\right\rangle$ for all $x, y \in C$ and $j=1,2, \ldots, M$. First, we see that $F\left(T_{r_{j}}^{f_{j}}\right)=\operatorname{EP}\left(f_{j}\right)=\operatorname{VI}\left(C, A_{j}\right)=F\left(T_{r_{j}}^{A_{j}}\right)$ for each $j=1,2, \ldots, M$.

Next, we show that $\left\{f_{j}\right\}_{j=1}^{M}$ satisfy conditions (A1)-(A4).

(A1) Consider $f_{j}(x, x)=\left\langle A_{j} x, x-x\right\rangle=0$ for all $x \in C$ and $j=1,2, \ldots, M$.

(A2) For each $x, y \in C$ and $j=1,2, \ldots, M$, we observe that

$$
\begin{aligned}
f_{j}(x, y)+f_{j}(y, x) & =\left\langle A_{j} x, y-x\right\rangle+\left\langle A_{j} y, x-y\right\rangle \\
& =\left\langle A_{j} x-A_{j} y, y-x\right\rangle .
\end{aligned}
$$

By the monotonicity of $A_{j}$, we obtain that $f_{j}$ is monotone. Thus $\left\{f_{j}\right\}_{j=1}^{M}$ satisfy condition (A2).

(A3) For each $x, y, z \in C$ and $j=1,2, \ldots, M$, we have by the continuity of $A_{j}$ that

$$
\begin{aligned}
\limsup _{t \downarrow 0} f_{j}(t z+(1-t) x, y) & =\underset{t \downarrow 0}{\lim \sup }\left\langle A_{j}(t z+(1-t) x), y-(t z+(1-t) x)\right\rangle \\
& =\left\langle A_{j} x, y-x\right\rangle \\
& =f_{j}(x, y) .
\end{aligned}
$$

This shows that $\left\{f_{j}\right\}_{j=1}^{M}$ satisfy condition (A3).

(A4) Let $u, v \in C$ and $s \in(0,1)$. Then, for each $x \in C$ and $j=1,2, \ldots, M$, we have

$$
\begin{aligned}
f_{j}(x, s u+(1-s) v) & =\left\langle A_{j} x, s u+(1-s) v-x\right\rangle \\
& =s\left\langle A_{j} x, u-x\right\rangle+(1-s)\left\langle A_{j} x, v-x\right\rangle \\
& =s f_{j}(x, u)+(1-s) f_{j}(x, v) .
\end{aligned}
$$

Thus $f_{j}$ is convex in the second variable. Let $u_{n} \in C$ and $\lim _{n \rightarrow \infty} u_{n}=u$. Then

$$
\begin{aligned}
f_{j}(x, u) & =\left\langle A_{j} x, u-x\right\rangle \\
& =\lim _{n \rightarrow \infty}\left\langle A_{j} x, u_{n}-x\right\rangle \\
& =\lim _{n \rightarrow \infty} f_{j}\left(x, u_{n}\right) .
\end{aligned}
$$

This shows that $f_{j}$ is lower semicontinuous in the second variable. Hence $\left\{f_{j}\right\}_{j=1}^{M}$ satisfy condition (A4). From Theorem 3.1 we obtain the desired result.

If we take $\alpha_{n}=0$ for all $n \in \mathbb{N}$ in Theorem 4.1, then we have the following corollary. 
Corollary 4.2. Let $E$ be a uniformly convex and uniformly smooth Banach space, and let $C$ be a nonempty, closed, and convex subset of $E$. Let $\left\{A_{j}\right\}_{j=1}^{M}$ be continuous and monotone operators from $C$ to $E^{*}$, and let $\left\{T_{i}\right\}_{i=1}^{\infty}$ be an infinitely countable family of closed and relatively quasi-nonexpansive mappings from $C$ into itself such that $F:=\left(\bigcap_{i=1}^{\infty} F\left(T_{i}\right)\right) \cap\left(\bigcap_{j=1}^{M} V I\left(C, A_{j}\right)\right) \neq \emptyset$. For any initial point $x_{0} \in E$ with $x_{1}=\Pi_{C_{1}} x_{0}$ and $C_{1}=C$, define the sequence $\left\{x_{n}\right\}$ as follows:

$$
\begin{gathered}
y_{n, i}=T_{i} x_{n}, \\
u_{n, i}=T_{r_{M, n}}^{A_{M}} T_{r_{M-1, n}}^{A_{M-1}} \cdots T_{r_{1, n}}^{A_{1}} y_{n, i}, \\
C_{n+1}=\left\{z \in C_{n}: \sup _{i \geq 1} \phi\left(z, u_{n, i}\right) \leq \phi\left(z, x_{n}\right)\right\}, \\
x_{n+1}=\prod_{C_{n+1}} x_{0}, \quad n \geq 1 .
\end{gathered}
$$

If $\lim \inf _{n \rightarrow \infty} r_{j, n}>0$ for each $j=1,2, \ldots, M$, then $\left\{x_{n}\right\}$ converges strongly to $\Pi_{F} x_{0}$.

Let $\varphi: C \rightarrow \mathbb{R}$ be a real-valued function. The convex minimization problem is to find that $\widehat{x} \in C$ such that

$$
\varphi(\widehat{x}) \leq \varphi(y) \quad \forall y \in C
$$

The solutions set of (4.9) is denoted by $\operatorname{CMP}(\varphi)$. For each $r>0$ and $x \in E$, define the mapping $T_{r}^{\varphi}: E \rightarrow C$ as follows:

$$
T_{r}^{\varphi}(x)=\left\{z \in C: \varphi(y)+\frac{1}{r}\langle y-z, J z-J x\rangle \geq \varphi(z), \forall y \in C\right\}
$$

Theorem 4.3. Let $E$ be a uniformly convex and uniformly smooth Banach space, and let $C$ be a nonempty, closed, and convex subset of $E$. Let $\left\{\varphi_{j}\right\}_{j=1}^{M}$ be lower semicontinuous and convex functions from $C$ to $\mathbb{R}$, and let $\left\{T_{i}\right\}_{i=1}^{\infty}$ be an infinitely countable family of closed and relatively quasinonexpansive mappings from $C$ into itself such that $F:=\left(\bigcap_{i=1}^{\infty} F\left(T_{i}\right)\right) \cap\left(\bigcap_{j=1}^{M} C M P\left(\varphi_{j}\right)\right) \neq \emptyset$. For any initial point $x_{0} \in E$ with $x_{1}=\Pi_{C_{1}} x_{0}$ and $C_{1}=C$, define the sequence $\left\{x_{n}\right\}$ as follows:

$$
\begin{gathered}
y_{n, i}=J^{-1}\left(\alpha_{n} J x_{n}+\left(1-\alpha_{n}\right) J T_{i} x_{n}\right), \\
u_{n, i}=T_{r_{M, n}}^{\varphi_{M}} T_{r_{M-1, n}}^{\varphi_{M-1}} \cdots T_{r_{1, n}}^{\varphi_{1}} y_{n, i}, \\
C_{n+1}=\left\{z \in C_{n}: \sup _{i \geq 1} \phi\left(z, u_{n, i}\right) \leq \phi\left(z, x_{n}\right)\right\}, \\
x_{n+1}=\prod_{C_{n+1}} x_{0}, \quad n \geq 1 .
\end{gathered}
$$


Assume that $\left\{\alpha_{n}\right\}$ and $\left\{r_{j, n}\right\}$ for $j=1,2, \ldots, M$ are sequences which satisfy conditions (B1) and (B2) of Theorem 3.1.

Then the sequence $\left\{x_{n}\right\}$ converges strongly to $\Pi_{F} x_{0}$.

Proof. Define $f_{j}(x, y)=\varphi_{j}(y)-\varphi_{j}(x)$ for all $x, y \in C$ and $j=1,2, \ldots, M$. Then $F\left(T_{r_{j}}^{f_{j}}\right)=$ $\operatorname{EP}\left(f_{j}\right)=C M P\left(\varphi_{j}\right)=F\left(T_{r_{j}}^{\varphi_{j}}\right)$ for each $j=1,2, \ldots, M$, and therefore $\left\{f_{j}\right\}_{j=1}^{M}$ satisfy conditions (A1) and (A2).

Next, we show that $\left\{f_{j}\right\}_{j=1}^{M}$ satisfy conditions (A3) and (A4). For each $x, y, z \in C$, we have by the lower semicontinuity of $\varphi_{j}$ that

$$
\begin{aligned}
\limsup _{t \downarrow 0} f_{j}(t z+(1-t) x, y) & =\underset{t \downarrow 0}{\lim \sup }\left(\varphi_{j}(y)-\varphi_{j}(t z+(1-t) x)\right) \\
& \leq \varphi_{j}(y)-\varphi_{j}(x) \\
& =f_{j}(x, y) .
\end{aligned}
$$

This implies that $\left\{f_{j}\right\}_{j=1}^{M}$ satisfy condition (A3).

Let $u, v \in C$ and $s \in(0,1)$. For each $x \in C$, we have by the convexity of $\varphi_{j}$ that

$$
\begin{aligned}
f_{j}(x, s u+(1-s) v) & =\varphi_{j}(s u+(1-s) v)-\varphi_{j}(x) \\
& \leq s \varphi_{j}(u)+(1-s) \varphi_{j}(v)-\varphi_{j}(x) \\
& =s\left(\varphi_{j}(u)-\varphi_{j}(x)\right)+(1-s)\left(\varphi_{j}(v)-\varphi_{j}(x)\right) \\
& =s f_{j}(x, u)+(1-s) f_{j}(x, v) .
\end{aligned}
$$

On the other hand, let $u_{n} \in C$ and $\lim _{n \rightarrow \infty} u_{n}=u$. By the lower semicontinuity of $\varphi_{j}$ we have

$$
\begin{aligned}
f_{j}(x, u) & =\varphi_{j}(u)-\varphi_{j}(x) \\
& \leq \liminf _{n \rightarrow \infty}\left(\varphi_{j}\left(u_{n}\right)-\varphi_{j}(x)\right) \\
& =\liminf _{n \rightarrow \infty} f_{j}\left(x, u_{n}\right) .
\end{aligned}
$$

Thus $\left\{f_{j}\right\}_{j=1}^{M}$ satisfy condition (A4). From Theorem 3.1 we also obtain the desired result.

If we take $\alpha_{n}=0$ for all $n \in \mathbb{N}$ in Theorem 4.3, then we have the following corollary.

Corollary 4.4. Let $E$ be a uniformly convex and uniformly smooth Banach space, and let $C$ be a nonempty, closed, and convex subset of $E$. Let $\left\{\varphi_{j}\right\}_{j=1}^{M}$ be lower semicontinuous and convex 
functions from $C$ to $\mathbb{R}$, and let $\left\{T_{i}\right\}_{i=1}^{\infty}$ be an infinitely countable family of closed and relatively quasinonexpansive mappings from $C$ into itself such that $F:=\left(\bigcap_{i=1}^{\infty} F\left(T_{i}\right)\right) \cap\left(\bigcap_{j=1}^{M} C M P\left(\varphi_{j}\right)\right) \neq \emptyset$. For any initial point $x_{0} \in E$ with $x_{1}=\Pi_{C_{1}} x_{0}$ and $C_{1}=C$, define the sequence $\left\{x_{n}\right\}$ as follows:

$$
\begin{gathered}
y_{n, i}=T_{i} x_{n}, \\
u_{n, i}=T_{r_{M, n}}^{\varphi_{M}} T_{r_{M-1, i}}^{\varphi_{M-1}} \cdots T_{r_{1, n}}^{\varphi_{1}} y_{n, i}, \\
C_{n+1}=\left\{z \in C_{n}: \sup _{i \geq 1} \phi\left(z, u_{n, i}\right) \leq \phi\left(z, x_{n}\right)\right\}, \\
x_{n+1}=\prod_{C_{n+1}} x_{0}, \quad n \geq 1 .
\end{gathered}
$$

If $\lim \inf _{n \rightarrow \infty} r_{j, n}>0$ for each $j=1,2, \ldots, M$, then $\left\{x_{n}\right\}$ converges strongly to $\Pi_{F} x_{0}$.

As a direct consequence of Theorem 3.1, we obtain the following application in a Hilbert space.

Theorem 4.5. Let $C$ be a nonempty, closed, and convex subset of a real Hilbert space $H$. Let $\left\{f_{j}\right\}_{j=1}^{M}$ be bifunctions from $C \times C$ to $\mathbb{R}$ which satisfies conditions $(A 1)-(A 4)$, and let $\left\{T_{i}\right\}_{i=1}^{\infty}$ be an infinitely countable family of closed and quasi-nonexpansive mappings from $C$ into itself such that $F:=\left(\bigcap_{i=1}^{\infty} F\left(T_{i}\right)\right) \cap\left(\bigcap_{j=1}^{M} E P\left(f_{j}\right)\right) \neq \emptyset$. For any initial point $x_{0} \in H$ with $x_{1}=P_{C_{1}} x_{0}$ and $C_{1}=C$, define the sequence $\left\{x_{n}\right\}$ as follows:

$$
\begin{gathered}
y_{n, i}=\alpha_{n} x_{n}+\left(1-\alpha_{n}\right) T_{i} x_{n}, \\
u_{n, i}=T_{r_{M, n}}^{f_{M}} T_{r_{M-1, n}}^{f_{M-1}} \cdots T_{r_{1, n}}^{f_{1}} y_{n, i}, \\
C_{n+1}=\left\{z \in C_{n}: \sup _{i \geq 1}\left\|z-u_{n, i}\right\| \leq\left\|z-x_{n}\right\|\right\}, \\
x_{n+1}=P_{C_{n+1}} x_{0}, \quad n \geq 1,
\end{gathered}
$$

where $P$ is the metric projection. Assume that $\left\{\alpha_{n}\right\}$ and $\left\{r_{j, n}\right\}$ for $j=1,2, \ldots, M$ are sequences which satisfy conditions (B1) and (B2) of Theorem 3.1.

Then the sequence $\left\{x_{n}\right\}$ converges strongly to $P_{F} x_{0}$.

Proof. Taking $E=H$ in Theorem 3.1, the result is obtained immediately.

Remark 4.6. Theorem 4.5 improves and extends the main results of [20-22] in the following senses:

(i) from the case of an equilibrium problem to a finite family of equilibrium problems;

(ii) from the class of nonexpansive mappings to the class of an infinitely countable family of quasi-nonexpansive mappings. 


\section{Acknowledgments}

The authors would like to thank Professor Simeon Reich and the referee for valuable suggestions on improving the manuscript and the Thailand Research Fund for financial support. The first author was supported by the Royal Golden Jubilee Grant PHD/0261/2551 and by the Graduate School, Chiang Mai University, Thailand.

\section{References}

[1] P. L. Combettes and S. A. Hirstoaga, "Equilibrium programming in Hilbert spaces," Journal of Nonlinear and Convex Analysis, vol. 6, no. 1, pp. 117-136, 2005.

[2] S.-y. Matsushita and W. Takahashi, "Weak and strong convergence theorems for relatively nonexpansive mappings in Banach spaces," Fixed Point Theory and Applications, no. 1, pp. 37-47, 2004.

[3] W. Takahashi and K. Zembayashi, "Strong convergence theorem by a new hybrid method for equilibrium problems and relatively nonexpansive mappings," Fixed Point Theory and Applications, vol. 2008, Article ID 528476, 11 pages, 2008.

[4] W. Takahashi, Nonlinear Functional Analysis: Fixed Point Theory and Its Applications, Yokohama Publishers, Yokohama, Japan, 2000.

[5] S. Reich, "A weak convergence theorem for the alternating method with Bregman distances," in Theory and Applications of Nonlinear Operators of Accretive and Monotone Type, A. G. Kartsatos, Ed., vol. 178 of Lecture Notes in Pure and Appl. Math., pp. 313-318, Marcel Dekker, New York, NY, USA, 1996.

[6] D. Butnariu, S. Reich, and A. J. Zaslavski, "Asymptotic behavior of relatively nonexpansive operators in Banach spaces," Journal of Applied Analysis, vol. 7, no. 2, pp. 151-174, 2001.

[7] D. Butnariu, S. Reich, and A. J. Zaslavski, "Weak convergence of orbits of nonlinear operators in reflexive Banach spaces," Numerical Functional Analysis and Optimization, vol. 24, no. 5-6, pp. 489-508, 2003.

[8] S. Kamimura and W. Takahashi, "Strong convergence of a proximal-type algorithm in a Banach space," SIAM Journal on Optimization, vol. 13, no. 3, pp. 938-945, 2002.

[9] P. Cholamjiak, "A hybrid iterative scheme for equilibrium problems, variational inequality problems, and fixed point problems in Banach spaces," Fixed Point Theory and Applications, vol. 2009, Article ID 719360, 18 pages, 2009.

[10] X. Qin, Y. J. Cho, S. M. Kang, and H. Zhou, "Convergence of a modified Halpern-type iteration algorithm for quasi- $\varphi$-nonexpansive mappings," Applied Mathematics Letters, vol. 22, no. 7, pp. 10511055, 2009.

[11] Y.Su, D. Wang, and M. Shang, "Strong convergence of monotone hybrid algorithm for hemi-relatively nonexpansive mappings," Fixed Point Theory and Applications, vol. 2008, Article ID 284613, 8 pages, 2008.

[12] K. Wattanawitoon and P. Kumam, "Strong convergence theorems by a new hybrid projection algorithm for fixed point problems and equilibrium problems of two relatively quasi-nonexpansive mappings," Nonlinear Analysis: Hybrid System, vol. 3, no. 1, pp. 11-20, 2009.

[13] Y. I. Alber, "Metric and generalized projection operators in Banach spaces: properties and applications," in Theory and Applications of Nonlinear Operators of Accretive and Monotone Type, A. G. Kartsatos, Ed., vol. 178 of Lecture Notes in Pure and Appl. Math., pp. 15-50, Marcel Dekker, New York, NY, USA, 1996.

[14] Ya. I. Alber and S. Reich, "An iterative method for solving a class of nonlinear operator equations in Banach spaces," Panamerican Mathematical Journal, vol. 4, no. 2, pp. 39-54, 1994.

[15] I. Cioranescu, Geometry of Banach Spaces, Duality Mappings and Nonlinear Problems, vol. 62 of Mathematics and Its Applications, Kluwer Academic Publishers, Dordrecht, The Netherlands, 1990.

[16] X. Qin, Y. J. Cho, and S. M. Kang, "Convergence theorems of common elements for equilibrium problems and fixed point problems in Banach spaces," Journal of Computational and Applied Mathematics, vol. 225, no. 1, pp. 20-30, 2009.

[17] E. Blum and W. Oettli, "From optimization and variational inequalities to equilibrium problems," The Mathematics Student, vol. 63, no. 1-4, pp. 123-145, 1994.

[18] W. Takahashi and K. Zembayashi, "Strong and weak convergence theorems for equilibrium problems and relatively nonexpansive mappings in Banach spaces," Nonlinear Analysis: Theory, Methods $\mathcal{E}$ Applications, vol. 70, no. 1, pp. 45-57, 2009. 
[19] F. Kohsaka and W. Takahashi, "Existence and approximation of fixed points of firmly nonexpansivetype mappings in Banach spaces," SIAM Journal on Optimization, vol. 19, no. 2, pp. 824-835, 2008.

[20] K. Nakajo and W. Takahashi, "Strong convergence theorems for nonexpansive mappings and nonexpansive semigroups," Journal of Mathematical Analysis and Applications, vol. 279, no. 2, pp. 372379, 2003.

[21] A. Tada and W. Takahashi, "Weak and strong convergence theorems for a nonexpansive mapping and an equilibrium problem," Journal of Optimization Theory and Applications, vol. 133, no. 3, pp. 359-370, 2007.

[22] W. Takahashi, Y. Takeuchi, and R. Kubota, "Strong convergence theorems by hybrid methods for families of nonexpansive mappings in Hilbert spaces," Journal of Mathematical Analysis and Applications, vol. 341, no. 1, pp. 276-286, 2008. 


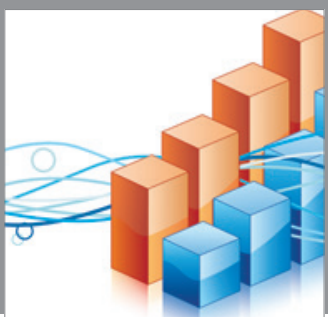

Advances in

Operations Research

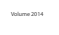

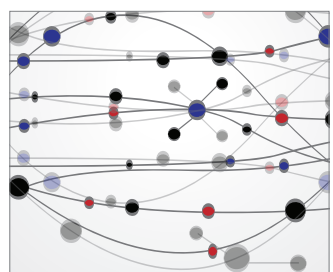

\section{The Scientific} World Journal
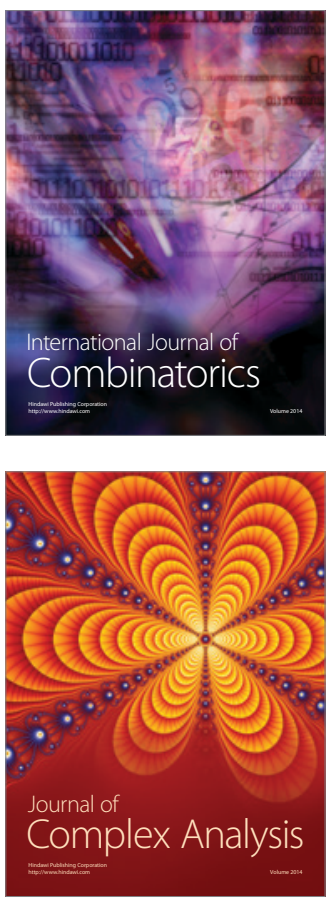

International Journal of

Mathematics and

Mathematical

Sciences
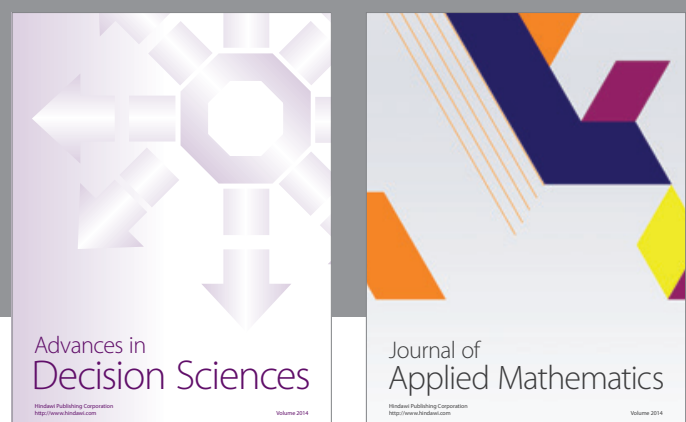

Journal of

Applied Mathematics
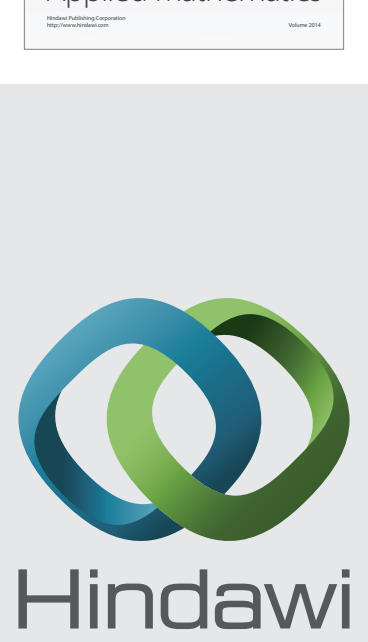

Submit your manuscripts at http://www.hindawi.com
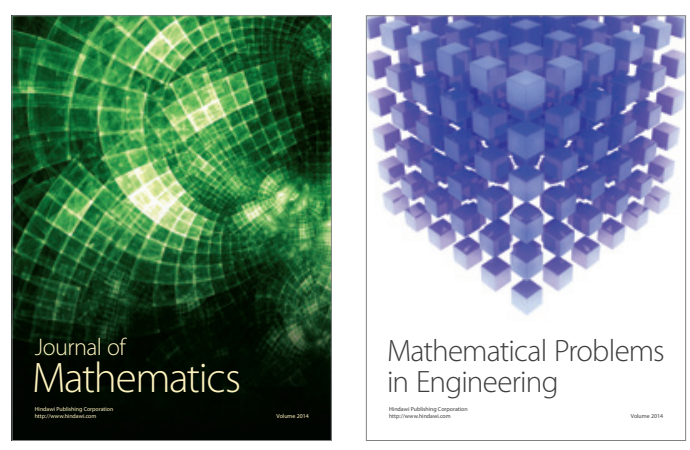

Mathematical Problems in Engineering
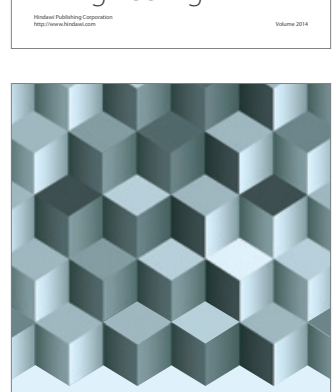

Journal of

Function Spaces
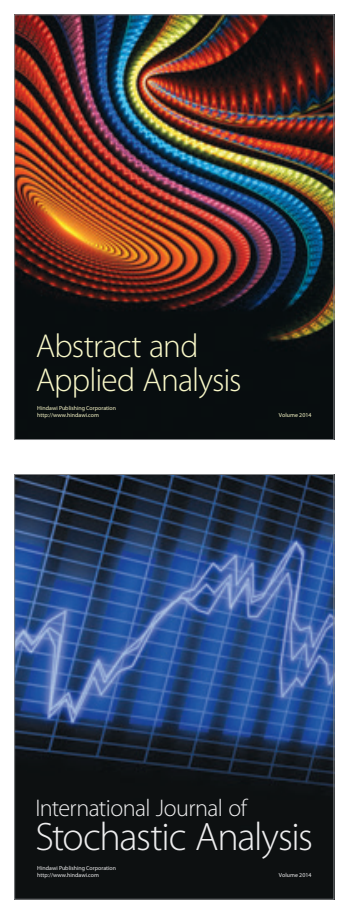

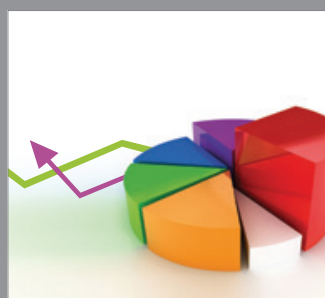

ournal of

Probability and Statistics

Promensencen
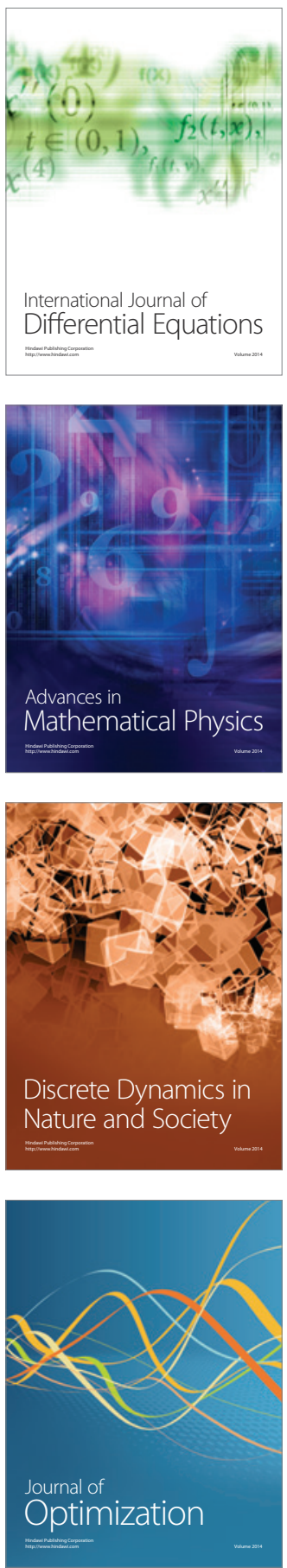